\title{
Les sentiments de justice à et sur l'école, Duru-Bellat Marie, Meuret Denis (dir.)
}

De Boeck, 2009, 276 pages

Pierre-Louis Gauthier

\section{OpenEdition}

\section{Journals}

Édition électronique

URL : https://journals.openedition.org/ries/799

DOI : $10.4000 /$ ries.799

ISSN : 2261-4265

Éditeur

France Education international

Édition imprimée

Date de publication : 1 décembre 2009

Pagination : 21-23

ISBN : 978-2-8542-0577-0

ISSN : 1254-4590

\section{Référence électronique}

Pierre-Louis Gauthier, "Les sentiments de justice à et sur l'école, Duru-Bellat Marie, Meuret Denis (dir.) », Revue internationale d'éducation de Sèvres [En ligne], 52 I décembre 2009, mis en ligne le 01 juillet 2011, consulté le 26 avril 2022. URL : http://journals.openedition.org/ries/799 ; DOI : https://doi.org/ $10.4000 /$ ries.799

Ce document a été généré automatiquement le 26 avril 2022

(C) Tous droits réservés 


\title{
Les sentiments de justice à et sur l'école, Duru-Bellat Marie, Meuret Denis (dir.)
}

De Boeck, 2009, 276 pages

\author{
Pierre-Louis Gauthier
}

\section{RÉFÉRENCE}

Duru-Bellat Marie, Meuret Denis (dir.), Les sentiments de justice à et sur l'école, De Boeck, 2009, $276 \mathrm{p}$.

1 L'ouvrage codirigé par Marie Duru-Bellat et Denis Meuret rassemble quatorze contributions présentées au séminaire du Réseau d'analyse des politiques publiques d'éducation (Dijon, 2007). Autour d'une problématique commune, l'ouvrage fédère des champs différents comme la psychologie sociale, l'économie, la sociologie de l'éducation, la sociologie de la santé.

2 Alliage subtil de composants dont le dosage varie au long de la scolarité, le sentiment de justice à l'école résulte d'une alchimie complexe. Fondé sur la croyance en un monde juste (Dalvert), l'émergence de ce sentiment s'opère à l'école, première institution sociale rencontrée par l'enfant au long de la maturation des capacités cognitives et des expériences morales (Bègue). Les élèves jugent de leur situation en fonction du principe d'égalité, lié au besoin d'être respecté, selon un contrat réciproque, même si la différence avec les adultes n'est pas remise en cause (Dubet). Un film récent illustre cette double perception ${ }^{1}$. Pourtant, les écarts (retards, absences, remarques désobligeantes ou blessantes) génèrent un soupçon qui augmente avec l'âge. Le principe d'égalité est pourtant la condition de la mesure du mérite, posée comme base du contrat scolaire. À partir de ce postulat, le problème glisse vers le contrôle et l'équité de la note, qui symbolise le système méritocratique. 
3 À l'enseignant de maintenir cet équilibre improbable entre l'égalité des élèves et la hiérarchie des mérites. Son autonomie doit lui permettre d'édicter, au sein même des contrôles, des normes qui prennent en compte les singularités et les héritages. L'instituteur du primaire y parvient généralement en une combinaison de critères «classants» et d'autres qui le sont moins. Dans le secondaire, la gageure est plus marquée car le mérite mesure "l'intelligence » et la naissance plutôt que l'effort. De plus, au sein des établissements, "les règlements intérieurs ne sont guère démocratiques et parfois même, les conseils de classe sont le contraire de la justice " (Dubet).

4 On est ainsi passé de l'élitisme républicain à l'égalité méritocratique des chances, d'un temps où la sélection était externalisée en amont de l'école à un temps où tout doit être géré au sein même de l'institution scolaire.

5 Plus clairement qu'avec la justice distributive en économie expérimentale (Trannoy), le rapprochement de l'éducation avec le secteur de la santé révèle une problématique semblable : généralisation et massification, inégalité médecins-patients et professeursélèves, mépris des pauvres (refus de consultation) et traitement scolaire des immigrés. Ici règnent également les inégalités sociales dont le décryptage, longtemps masqué par la notion de "groupes à risques » (cas des maladies professionnelles), a été plus tardif. Les conclusions sont identiques: l'égalité d'accès à la santé ne suffit pas à assurer l'égalité des chances de guérison, la réponse juste étant une répartition inégalitaire des moyens de santé suivant les groupes, qui s'apparenterait à la discrimination positive en éducation (Visier).

6 La dimension internationale de l'ouvrage permet de replacer la perception du méritebesoin-égalité dans un contexte comparatif et de rappeler que le remède aux inégalités ne se situe pas dans un égalitarisme absolu à la Babeuf, mais dans un équilibre entre plusieurs exigences et besoins de base (Forsé, Parodi).

7 Historiquement, la notion de justice s'est diffusée à travers les politiques éducatives et les structures (Dupriez et Dumay). Les étapes successives ont été l'accès à l'éducation, puis à l'égalité des chances et de traitement. Que l'on songe au progrès considérable de la création de l'école primaire pour tous. Actuellement, si l'école peine à établir l'égalité des parcours scolaires, c'est que le mérite et le talent, dont on connait la relativité, restent les seuls critères. Le principe de l'égalité des chances s'est affaibli puisqu'il s'agit uniquement d'égalité de traitement et que les dispositifs compensatoires, conçus comme une formation additionnelle, restent faibles. La méritocratie ${ }^{2}$, érigée en principe idéologique, légitime la hiérarchisation des groupes sociaux et « on peut s'interroger sur la prévalence de la méritocratie scolaire » (Duru-Bellat et Brinbaum). Les effets contradictoires du diplôme " consécrateur » vs effet libérateur de l'éducation varient selon les pays (Tenret) et l'enquête menée dans les écoles francophones de Belgique montre des conceptions erratiques de l'égalité (Dupriez, Dumay).

La distribution des publics au sein des établissements reste l'une des sources du sentiment d'injustice. Elle résulte de toute une stratégie d'évitement des parents qui ne se sentent ni partenaires ni acteurs du système. Ils se trouvent placés devant le dilemme du "bon citoyen " favorable à la mixité sociale versus le «bon parent» désireux de donner à son enfant un cadre " homogène " (de niveau, ethnique, social ?). Cette volonté de l'« en soi » est justifiée par le refus de la logique sacrificielle de la sectorisation (Zoïa). 
9 Au fil des contributions, l'ouvrage décline la problématique à tous les niveaux de la scolarisation, contrairement à maintes recherches qui limitent leurs travaux aux étages «nobles» des enseignements secondaire et supérieur. Il donne un peu plus la parole aux élèves, au travers d'entretiens de groupe qui révèlent leur argumentation et les variations dans leurs jugements (Caillet). Des mises en situation se rapportant à la justice professorale éclairent les stratégies destinées à se protéger de «la violence du principe de mérite" (Richardot et Lautier). L'élève français, comparativement à d'autres, exprime fortement le sentiment d'injustice ressenti à l'école et son origine, la relation avec les enseignants (Desvignes, Meuret).

Ici s'ouvre une brèche dans le contrat social de l'école: les enseignants croient-ils toujours à l'égalité en éducation? Envisagent-ils d'atténuer les différences de résultats entre les groupes sociaux? Leur conception ontologique de l'égalité et du respect de tous les élèves est une conception modeste, sinon naïve, de l'égalité.

11 L'ouvrage marque un jalon important dans la recherche en l'éducation. La problématique du besoin de justice examinée au long de la scolarité obligatoire à partir de plusieurs champs disciplinaires, la dimension internationale et comparative lui donnent une résonance inhabituelle dans les travaux actuels. Enfin, l'audace et l'impertinence du propos par rapport à l'antienne traditionnelle sur les inégalités scolaires font honneur aux auteurs.

\section{NOTES}

1. Entre les murs de Laurent Cantet (2008).

2. Michaël Young, The Rise of Meritocracy (1958).

\section{AUTEURS}

\section{PIERRE-LOUIS GAUTHIER}

Pierre-Louis Gauthier est Inspecteur d'académie (H), a été professeur d'histoire-géographie, inspecteur de l'éducation nationale, directeur d'école normale, directeur adjoint d'IUFM. Il est membre du comité de rédaction de la Revue internationale d'éducation de Sèvres depuis 1994, pour laquelle il a notamment coordonné les numéros sur la formation des enseignants ( $n^{\circ} 20$ et 21$)$, les diplômes et les certifications ( $\left.n^{\circ} 37\right)$, et sur l'école primaire $\left(n^{\circ} 41\right)$. 